\title{
Direct Observation of Electron Heating by Electron Landau Damping of Alfvén Ion Cyclotron Waves Using Thomson Scattering System in the Tandem Mirror GAMMA 10/PDX ${ }^{*)}$
}

\author{
Masayuki YOSHIKAWA, Ryuya IKEZOE, Koichi OHTA, Xiaolong WANG, Junko KOHAGURA, \\ Yoriko SHIMA, Masayuki CHIKATSU, Kazuya ICHIMURA, Mizuki SAKAMOTO, \\ Makoto ICHIMURA, Tsuyoshi IMAI, Ryutaro MINAMI, \\ Kohei TSUMURA and Yousuke NAKASHIMA \\ Plasma Research Center, University of Tsukuba, Tsukuba, Ibaraki 305-8577, Japan
}

(Received 16 November 2015 / Accepted 9 March 2016)

\begin{abstract}
In the hot ion mode of the tandem mirror GAMMA 10/PDX experiments, increases in the flux and temperature of end-loss electrons were observed after the excitation of Alfvén ion cyclotron (AIC) modes. The increase of the electron temperature in the central cell is directly observed using a Thomson scattering system, confirming electron heating due to the electron Landau damping of AIC modes.
\end{abstract}

(C) 2016 The Japan Society of Plasma Science and Nuclear Fusion Research

Keywords: GAMMA 10/PDX, Thomson scattering, electron temperature, Alfvén ion cyclotron wave

DOI: $10.1585 /$ pfr. 11.2402051

\section{Introduction}

In the tandem mirror GAMMA 10/PDX, ion cyclotron range of frequency (ICRF) waves have been used for plasma production, heating, and sustaining magnetohydrodynamic stability $[1,2]$. A maximum ion temperature of $10 \mathrm{keV}$ and a temperature anisotropy (defined as the temperature ratio between directions perpendicular, $T_{\perp}$, and parallel, $T_{/ /}$, to the magnetic field line) of more than 10 have been achieved. In such a strong temperature anisotropy condition, slow Alfvén ion cyclotron (AIC) waves were spontaneously excited in the central cell of GAMMA 10/PDX [3-6]. The AIC mode is normally observed using magnetic probes [7] and reflectometers [8,9]. Diamagnetism increases with increased power of heating, and the anisotropy becomes strong. Saturation of the diamagnetism is observed in some experiments. The excitation of AIC modes that can scatter hot ions confined in the magnetic mirror field into the loss cone of the velocity space in the central cell is a possible confinement degradation mechanism. On the other hand, the parametric decay of heating ICRF waves was discussed for the saturation mechanism of diamagnetism [4]. Low-frequency magnetic fluctuations with beat frequencies between heating ICRF waves and discrete peaks of AIC modes were clearly detected in GAMMA 10/PDX along with different frequencies of AIC modes [10].

AIC modes exhibit several discrete peaks in the frequency spectrum just below the ion cyclotron frequency. Although no resonance with ions has been observed, res-

author'se-mail: yosikawa@prc.tsukuba.ac.jp

*) This article is based on the presentation at the 25th International Toki Conference (ITC25). onant interaction with electrons can be extracted. Accelerated electrons at the machine end of GAMMA 10/PDX were directly detected, and the effects of AIC modes on electrons were examined using the loss electron energy (LEE) analyzer [5,6]. An AIC mode leads to increases in the end loss electron flux and electron temperature that were measured using the LEE analyzer. Based on the analysis of the transient feature of the end loss electron temperature, this interaction was attributed to electron Landau damping [6]. However, electron temperatures measured using the LEE analyzer may include the effects of the potential difference between the central and end regions.

In this study, the first direct observation of electron heating by the interaction of AIC waves is reported. Electron temperatures were directly measured using a Thomson scattering system with changing laser injection time every $10 \mathrm{~ms}$ per shot. This is unlike the previous reports of refs. 5 and 6 , where electron temperatures were estimated indirectly using LEE signals.

\section{Experimental Setup}

The tandem mirror GAMMA 10/PDX consists of a central cell, two anchor cells located at both ends of the central cell, and two mirror cells connected to the anchor cells. Detailed description of GAMMA 10/PDX has been provided elsewhere [1-12]. The magnetic field strength at the midplane of the central cell is $0.405 \mathrm{~T}$, and the mirror ratio of the central cell is 5. A segmented limiter with a diameter of $0.36 \mathrm{~m}$ is set near the midplane. Plasma is produced by applying RF1 $(9.9-10.3 \mathrm{MHz})$ in combination with short-pulse-gun-produced plasmas from both 


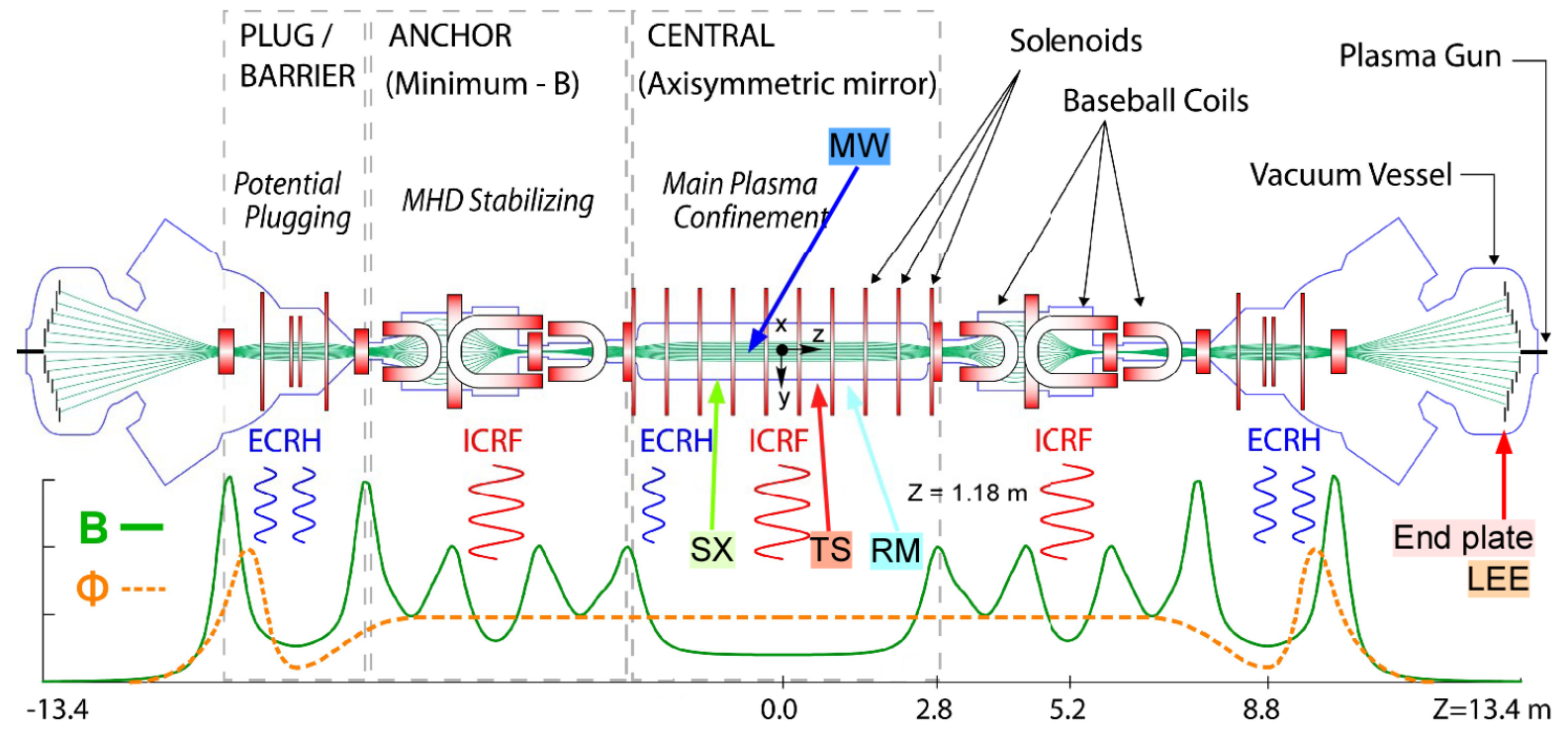

Fig. 1 Schematic of GAMMA 10/PDX.

ends and hydrogen gas injection near RF antennas. Moreover, plasma is heated by applying RF2 $(\sim 6.36 \mathrm{MHz})$ with a fundamental ion-cyclotron resonance layer near the midplane of the central cell. The RF1 frequency is selected to be a fundamental ion-cyclotron resonance frequency near the midplane of an anchor cell. Two types of antennas are installed at both (east and west) ends of the central cell. Figure 1 shows a schematic of the GAMMA 10 coil system and magnetic field profile in the axial direction. The $x$ and $y$ axes are perpendicular to the magnetic field in the vertical and horizontal directions, respectively. The $z$ axis is parallel to the magnetic field.

A microwave reflectometer (RM) was constructed for studying high-frequency fluctuations in the plasma core region [9]. This system consists of two simple heterodyne reflectometers. The relevant microwave frequency range is $8.2-12.4 \mathrm{GHz}$ ( $\mathrm{X}$ band), which covers a wide range of GAMMA 10/PDX plasma $\left(n_{\mathrm{e}} \sim 2 \times 10^{12} \mathrm{~cm}^{-3}\right)$.

Plasma touches the end plates electrodes along the magnetic field. End plates are made of stainless steel and are located at both ends of GAMMA 10/PDX [10]. These plates consist of several plates that are radially divided into five sections and are connected to the ground though a cement resistor. We measured the floating potential of each end plate using a $50-\Omega$ pick-up resistor set between the 1.111-M $\Omega$ resistor and ground by a high-speed analog-todigital converter $(1 \mathrm{MSa} / \mathrm{s})$. The floating potential is usually negative.

Several microwave interferometers for density measurements are present in GAMMA 10/PDX [12]. A movable microwave interferometer is set at $z=-60 \mathrm{~cm}$ for electron line density radial profile measurements, and its movable horn position is changed shot-by-shot. We can obtain electron density radial profiles in the $y$ direction using the Abel transform technique.
To measure the electron temperature, we installed a yttrium-aluminum-garnet Thomson scattering system at $z=60 \mathrm{~cm}$ for the radial measurement in the $x$ direction $[13,14]$. This system can measure the electron temperature and density at several radial positions in a single period for single laser and plasma shots. Unfortunately, we could not obtain the radial profile of the electron temperature and density in this experiment. The Thomson scattering system measures the perpendicular component of the electron temperature. In GAMMA 10/PDX, the electron collision time is less than $10 \mu \mathrm{s}$ and the electron temperature is normally isotropic.

We measure the radial profiles of soft X-ray (SX) intensities using two SX diagnostic system sets constructed using a 16-channel semiconductor X-ray detector (XSD) array [15]. Two XSD arrays separated by $135^{\circ}$ in the azimuthal direction are placed at $z=-120 \mathrm{~cm}$.

End-loss electrons are measured using a multigridtype LEE analyzer installed on the end wall of the vacuum vessel [5,6]. End-loss electrons enter the analyzer through a small hole on the electrically floating end plate located in front of the end wall. The collector current of the analyzer represents the electron current flowing into the end plate. The electron repeller voltage of the analyzer is swept, and current-voltage characteristics of the end loss electrons can be obtained in one shot. The LEE analyzer measures the parallel component of the electron temperature.

\section{Experiments}

Plasma is heated and maintained by applying ICRF waves; RF1 is applied for $t=51-240 \mathrm{~ms}$, and RF2 is applied for $t=53-240 \mathrm{~ms}$. The rise time of RF2 in these experiments is set to $90 \mathrm{~ms}$. RF3 is applied for $t=190-240 \mathrm{~ms}$. Figure 2 (a) shows the electron line den- 


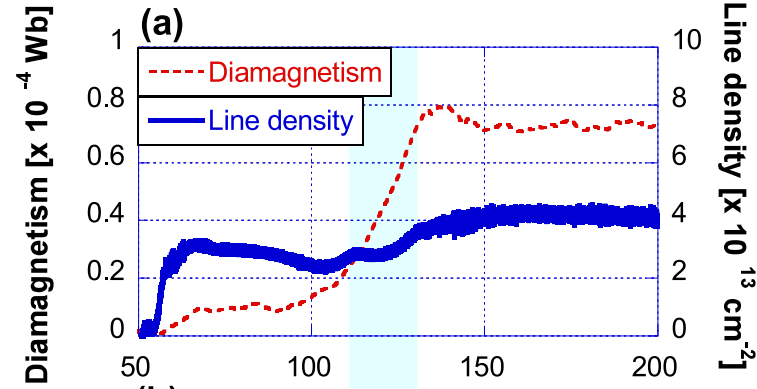

(b)
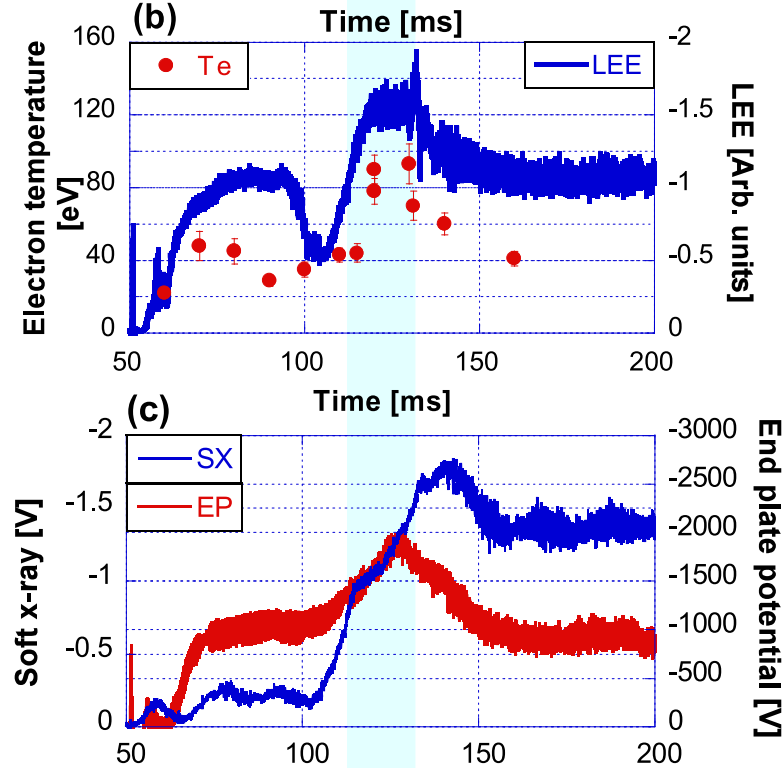

(d)

Time $[\mathrm{ms}]$

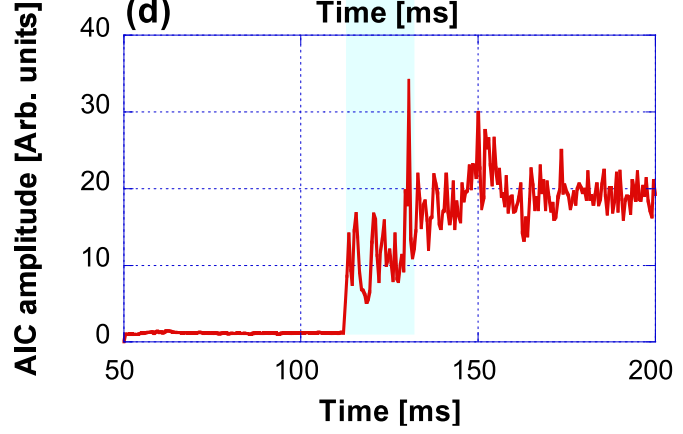

Fig. 2 (a), (b), (c) and (d) show diamagnetism and line density, electron temperature and end loss electron current (LEE), soft X-ray intensity (SX) and end plate potential (EP), and AIC amplitude, respectively.

sity (blue solid line) and diamagnetism (red dotted line). The time evolution of the electron temperature measured using the Thomson scattering system for different laser injection times shot-by-shot is shown in Fig. 2 (b) together with the end loss electron current obtained using the LEE analyzer. Figure 2 (c) shows the SX intensity and end plate \#1 potential (EP), and Fig. 2 (d) shows the AIC amplitude measured via reflectometry. Diamagnetism increases with increased electron temperature from $t=100 \mathrm{~ms}$ to $130 \mathrm{~ms}$. The maximum electron temperature value at $t=120$ $130 \mathrm{~ms}$ is obtained faster than the maximum diamagnetism value at $t=140 \mathrm{~ms}$. The electron line density slightly

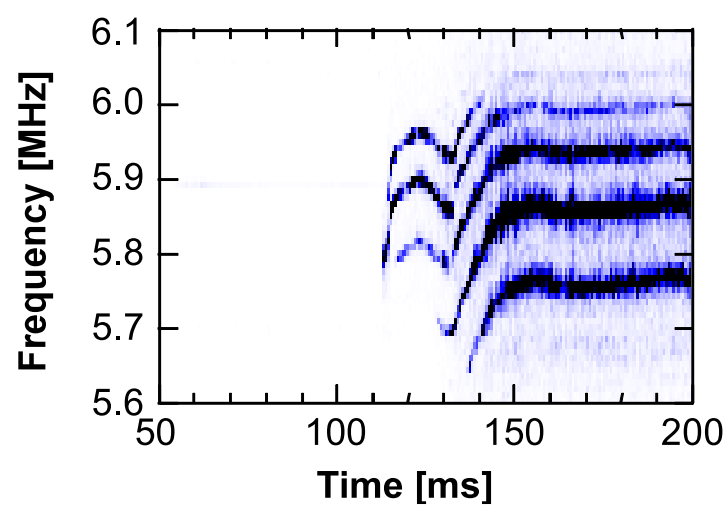

Fig. 3 AIC wave spectra measured using the RM system.

increases from $t=115 \mathrm{~ms}$ to $130 \mathrm{~ms}$ (light blue hatched region in Fig. 2). The line density then further increased until $t=150 \mathrm{~ms}$. The maximum value of the SX intensity is obtained at approximately $t=140 \mathrm{~ms}$, which is almost identical to the time of maximum diamagnetism. The maximum value of the EP potential is obtained at approximately $t=125 \mathrm{~ms}$, prior to the time of maximum diamagnetism. Together with the increase of the AIC fluctuation intensity in the light blue hatched region of Fig. 2, the electron temperature also increases with the AIC amplitude up to $90 \mathrm{eV}$. The electron temperature then decreases to about half of the maximum value. High AIC amplitudes from $t=140 \mathrm{~ms}$ correspond to decreasing electron temperature as well as decreases in diamagnetism, LEE, and EP values. Changes in the SX intensity follow changes in diamagnetism values.

In Fig. 3, we show the AIC spectrum obtained using the RM system. For $t=115-130 \mathrm{~ms}$, AIC waves were clearly observed at three frequencies and show a frequency peak at approximately $t=120 \mathrm{~ms}$. Starting at $t=135 \mathrm{~ms}$, the startups of the frequency of AIC waves are observed again.

\section{Discussion}

An AIC mode is excited when a drive term $\beta\left(T_{\perp} / T_{/ /}\right)^{2}$ exceeds a threshold value [16]. In GAMMA 10/PDX, the value of $\beta$ is equal to a few percent. Usually, the AIC mode exhibits several peaks in the frequency spectrum. As the diamagnetic signal increases at about $0.3 \times 10^{-4} \mathrm{~Wb}$, an AIC mode is excited. The electron temperature increases significantly with the excitation of the AIC mode but gradually decreases with time. Without the AIC wave, the electron temperature increases with the electron drag power on the hot ions. Figure 4 shows the relationship between $T_{\mathrm{e}}$ and $n_{\mathrm{e}} D / T_{\mathrm{e}}^{1.5}[5,6]$ and the electron drag line. Here, $n_{\mathrm{e}} D / T_{\mathrm{e}}^{1.5}$ is proportional to the electron drag power, and $n_{\mathrm{e}}$, $T_{\mathrm{e}}$ and $D$ represent the electron density, electron temperature and diamagnetism, respectively. The electron drag line is obtained by linear fitting of $T_{\mathrm{e}}$ versus $n_{\mathrm{e}} D / T_{\mathrm{e}}^{1.5} \mathrm{ex}-$ cept for the data collected for $t=70-80 \mathrm{~ms}$ and AIC heat- 


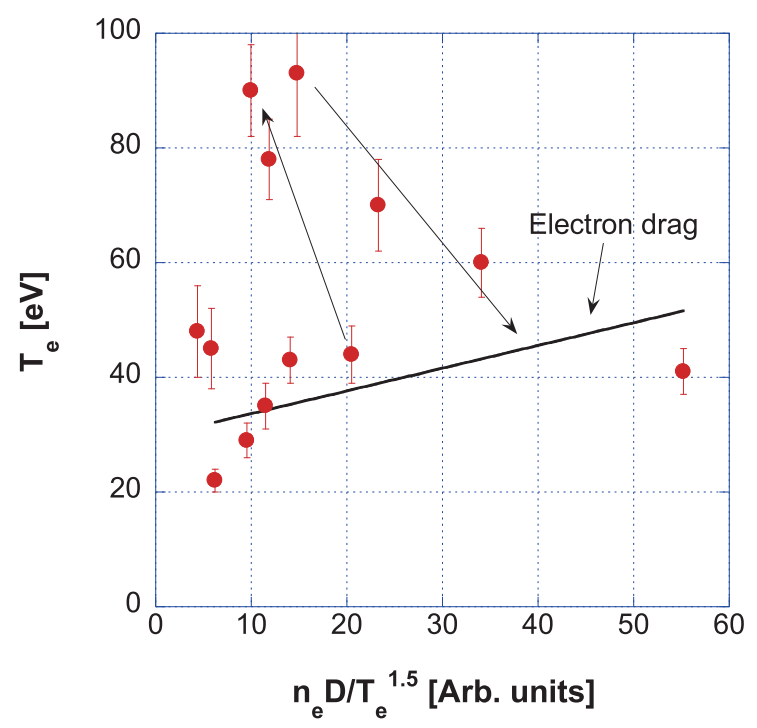

Fig. $4 T_{\mathrm{e}}$ versus $n_{\mathrm{e}} D / T_{\mathrm{e}}^{1.5}$. Electron drag is obtained by linear fitting from $t=60$ to $110 \mathrm{~ms}$ and $t=160 \mathrm{~ms}$, except for $t=70$ and $80 \mathrm{~ms}$ and AIC heated periods.

ing periods of $t=115-130 \mathrm{~ms}$. Some of the $T_{\mathrm{e}}$ values at $t<110 \mathrm{~ms}$ are due to the direct heating of electrons by the $\mathrm{RF}$ electric field. The significant increase of electron temperature with the start of AIC mode condition for $t=115$ $130 \mathrm{~ms}$ is clearly observed. The electron temperature decreases gradually for $t=130-160 \mathrm{~ms}$. The mechanism of the increased direct electron heating by the AIC mode is thought to be due to electron Landau damping $[5,6]$. The electron temperatures of electron drag by the hot ions and from AIC waves are about $40 \mathrm{eV}$ and $90 \mathrm{eV}$, respectively. Unfortunately, $T_{\mathrm{e}}$ values measured using the LEE analyzer could not be obtained. This makes it difficult to determine the anisotropy of the electron temperature. As the driving term $\beta\left(T_{\perp} / T_{/ /}\right)^{2}$ increases, the AIC mode changes its character from a traveling wave to a standing wave like and the absorption power of the electron Landau damping decreases [6]. This is the origin of the gradual decrease in the electron temperature to the level expected for conditions in the presence of the AIC wave excitation when only the drag power effect is present after reaching the maximum value of electron temperature.

Figures 5 (a) and 5 (b) show the radial profiles of electron densities and SX intensities, respectively, at $t=100$, 130 , and $160 \mathrm{~ms}$. Electron density radial profiles were measured using the microwave interferometer system. The full widths at half maximums (FWHMs) of electron density radial profiles are almost constant during the AIC mode. The FWHM of the SX intensity radial profile at $t=160 \mathrm{~ms}$ is larger than those at $t=100 \mathrm{~ms}$ and $130 \mathrm{~ms}$. Moreover, the FWHMs of these profiles at $t=100$ and $130 \mathrm{~ms}$ are almost the same. The SX intensity at the core region is mainly increased at $t=130 \mathrm{~ms}$. Comparison of the radial profiles of the electron density and the SX intensity shows that the AIC mode electron temperature heating

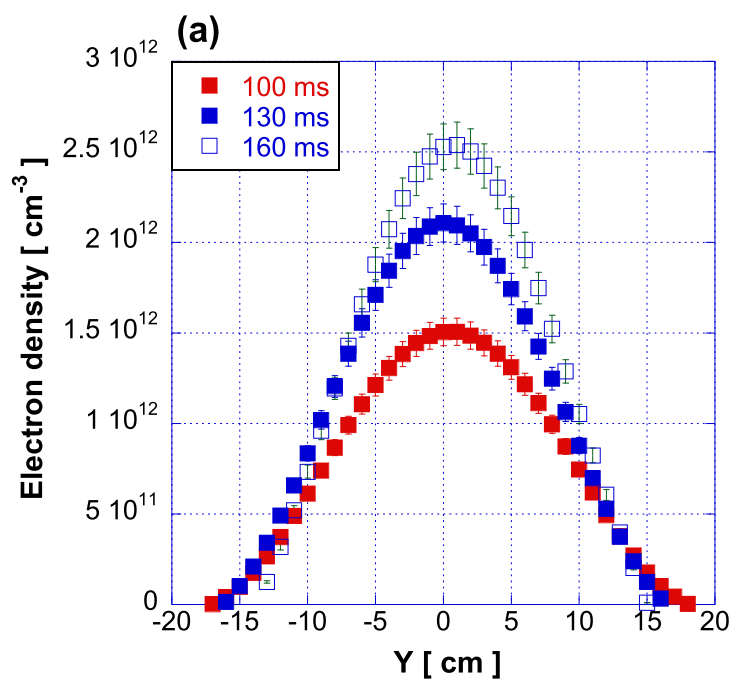

(b)

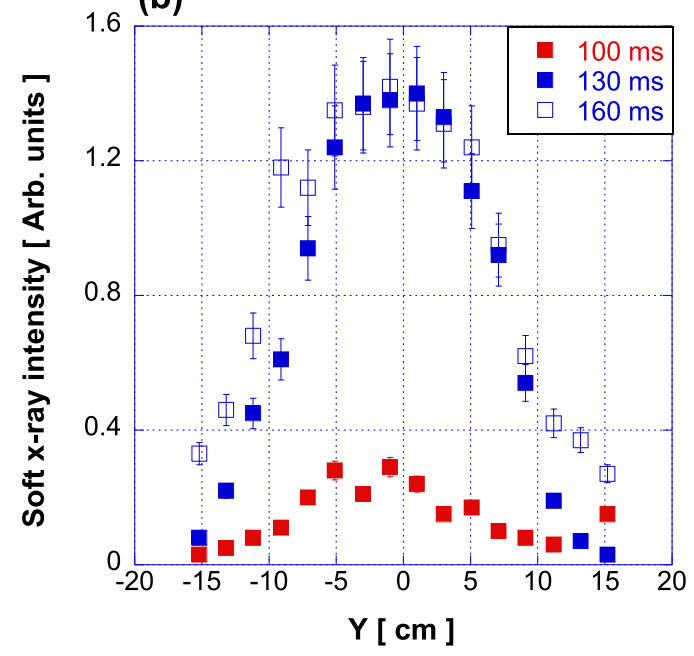

Fig. 5 Radial profiles of electron densities (a) and soft x-ray intensities (b) at $t=100,130$, and $160 \mathrm{~ms}$, respectively.

is carried out in the core plasma region.

\section{Summary}

We carried out the direct observations of electron heating by the AIC wave interaction using the Thomson scattering system. The electron temperature increases up to about $90 \mathrm{eV}$ at the initial excitation phase of the AIC waves when the Landau damping condition is satisfied. Using the Thomson scattering system, the electron temperature behavior in the central cell was directly observed and the previously reported electron heating by Landau damping of the AIC waves was confirmed.

\section{Acknowledgments}

The authors thank the members of the GAMMA 10 group of the University of Tsukuba for their collaboration. This study was conducted with the support and under the auspices of the NIFS Collaborative Research Program (NIFS11KUGM056) and the Bidirectional 
Collaboration Research Programs (NIFS14KUGM086, NIFS14KUGM088).

[1] Y. Nakashima et al., Fusion Sci. Technol. 68, 28 (2015).

[2] M. Ichimura et al., Plasma Phys. Rep. 28, 727 (2002).

[3] M. Ichimura et al., Phys. Rev. Lett. 70, 2734 (1993).

[4] M. Ichimura et al., J. Plasma Fusion Res. SERIES 8, 893 (2009).

[5] T. Saito et al., Phys. Rev. Lett. 82, 1169 (1999).

[6] T. Saito et al., J. Phys. Soc. Jpn. 72, 1977 (2003).
[7] A. Kumagai et al., Jpn. J. Appl. Phys. 36, 6778 (1997).

[8] A. Mase et al., Phys. Fluids B5, 1677 (1993).

[9] R. Ikezoe et al., Nucl. Fusion 53, 073040 (2013).

[10] R. Ikezoe et al., Phys. Plasmas 22, 090701 (2015).

[11] M. Yoshikawa et al. Nucl. Fusion 53, 073031 (2013).

[12] M. Yoshikawa et al., Trans. Fusion Sci. Technol. 55, 19 (2009).

[13] M. Yoshikawa et al., J. Instrum. 7, C03003 (2012).

[14] M. Yoshikawa et al., J. Instrum. 8, C10016 (2013).

[15] R. Minami et al., Rev. Sci. Instrum. 85, $11 \mathrm{D} 807$ (2014).

[16] R. Katsumata et al., Phys. Plasmas 3, 4489 (1996). 